\title{
Artificial Light at Night Increases Aedes aegypti Mosquito Biting Behavior with Implications for Arboviral Disease Transmission
}

\author{
Samuel S. C. Rund, ${ }^{1,2}$ Laura F. Labb, ${ }^{1,2}$ Owen M. Benefiel, ${ }^{1,2}$ and Giles E. Duffield ${ }^{1,2 *}$ \\ ${ }^{1}$ Department of Biological Sciences, Galvin Life Science Center, University of Notre Dame, Notre Dame, Indiana; ${ }^{2}$ Eck Institute for Global Health, \\ University of Notre Dame, Notre Dame, Indiana
}

\begin{abstract}
Aedes aegypti mosquito is a major vector of arboviral disease. Here, we report that the biting behavior of normally daytime active anthropophilic Ae. aegypti mosquitoes on human hosts is abnormally increased at night following exposure to artificial light at night (ALAN). Biting was examined using a human host assay where caged mosquitoes were exposed to a human arm and blood-feeding measured. Mosquitoes were tested during the daytime, nighttime, or challenged with ALAN. As predicted from the Ae. aegypti diel/circadian biting cycle, maximal biting occurred during daytime and lowest level occurred at night. Biting in the ALAN group was increased compared with time-matched nighttime controls. These data reveal that exposure to ALAN increases nocturnal blood-feeding behavior. This finding highlights the concern that globally increasing levels of light pollution could be impacting arboviral disease transmission, such as dengue fever and Zika, and has implications for application of countermeasures for mosquito vector control.
\end{abstract}

Arthropod-borne arboviruses, such as those of which the anthropophilic Aedes aegypti mosquito is the primary vector, remain a continuous threat to human health. These include dengue and yellow fever, which cause significant annual morbidity and mortality throughout the tropics and subtropics, ${ }^{1}$ whereas Zika and chikungunya virus are rapidly emerging diseases threatening new geographic areas around the world. ${ }^{1}$ The growing incidence of insecticide resistance, a lack of effective vaccines or specific medications against most arboviruses, and an increasing mosquito geographical range as a result of climate change, changes in land use, and increased global travel highlight the need for a greater understanding of Ae. aegypti physiology to combat these diseases. ${ }^{2}$

Aedes aegypti is daytime active (diurnal), expressing both a pronounced bout of flight activity and biting behavior in the early morning and followed by a second and longer window of activity that occurs during the afternoon. This activity terminates during or at the end of dusk. Generally, no or little flight activity or biting occurs during the night. ${ }^{3,4}$

Artificial light at night (ALAN) (i.e., "light pollution") is increasing in both the quantity of light and proportion of the population affected globally, and this phenomenon is occurring in both developed and developing countries. ${ }^{5}$ This presents significant ecological and human and animal health implications. ${ }^{6-8}$ Light can have profound effects on the physiology and behavior of animals, including insects, shaping and tuning behaviors that change over 24 hours. This is through entrainment of the endogenous circadian clock and by directly driving genetic and behavioral responses (i.e., masking) that include phototaxic and photophobic responses. $^{4,7,9-14}$ In mosquitoes specifically, they exhibit responses to changes in sunlight and moonlight, resulting in modifications to their flight activity including swarming, and in host-seeking and blood-feeding behaviors. ${ }^{4,11,14,15}$

The objective of this study was to examine the effect of ALAN on Ae. aegypti biting behavior. We hypothesized that exposure to a low level of white light of fixed duration at

${ }^{*}$ Address correspondence to Giles E. Duffield, Department of Biological Sciences, Galvin Life Science Center, University of Notre Dame, Notre Dame, IN 46556. E-mail: duffield.2@nd.edu night would increase the biting activity of female Ae. aegypti mosquitoes.

Aedes aegypti mosquitoes ${ }^{16}$ were reared and maintained in the laboratory at $26 \pm 1{ }^{\circ} \mathrm{C}$ and $85 \% \pm 1 \%$ humidity on a $12: 12$ light-dark (LD) cycle (11 hours 250 lux full light, 11 hours darkness, and 1 hour dawn and dusk transitions; Figure 1A). Access to fructose was provided ad libitum. Time was measured in Zeitgeber time (ZT), with ZT12 marking the time of lights off and the start of the night phase of the LD cycle. Zeitgeber time 0 marks the start of the daytime at the end of dawn transition. Female mosquito biting activity was examined at two different times of the photoschedule, first at 2 hours before the time of lights off (ZT10; late afternoon) and then at 4 hours after lights off (ZT16; middle of the night) (Figure 1B). Biting activity was assessed using a human arm assay using a standardized protocol. ${ }^{11}$ Populations of 30 mated, but not blood-fed, 5-9 days post-eclosion female mosquitoes were held in pint-sized pots provided with a mesh lid, allowing them transient access to a human host (Investigator's arm) and to blood feed. Mosquitoes were arranged in pots at least 24 hours before experimentation. The mosquitoes were exposed to the arm for 6 minutes while in a dark room, and the number of mosquitoes taking a blood feed was counted. Mosquitoes were anesthetized using $\mathrm{CO}_{2}$ and assessed under the microscope for the presence of blood in the abdomen. A third study group was subjected to a $30-$ minute treatment with white light (50 lux, incandescent lamp) at the specific time of ZT15.5, and biting activity assessed 30 minutes later in the dark at ZT16 (middle of the night) (Figure 1B). To prevent human disturbance of the mosquitoes, manipulation of lighting conditions was automated and conducted remotely. These three groups allowed assessment of the propensity of the mosquitoes to bite during their normal preferred biting time of the late afternoon (ZT10) and at a time of the 24-hour diel cycle when they would be expected to show low level biting activity, that is, during the middle of the night (ZT16). The manipulated group were provided with ALAN and assessed also in the middle of the night at ZT16. The experiment was repeated five times. The study was approved by the University of Notre Dame Office of Research Compliance Institutional Review Board (IRB) (17-05-3891), and informed consent was received from individuals participating in the experiments. 


\section{A Light Dark Cycle/Photoschedule}

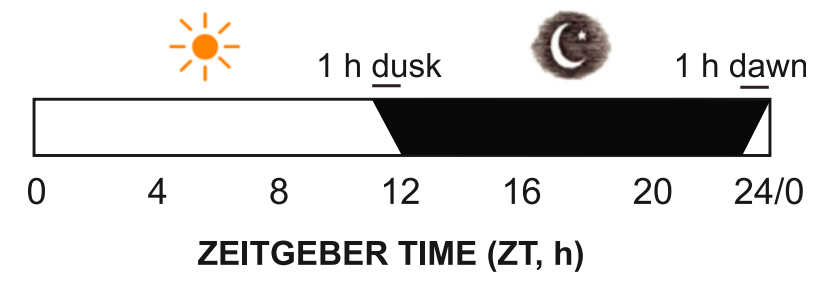

B Experimental Design

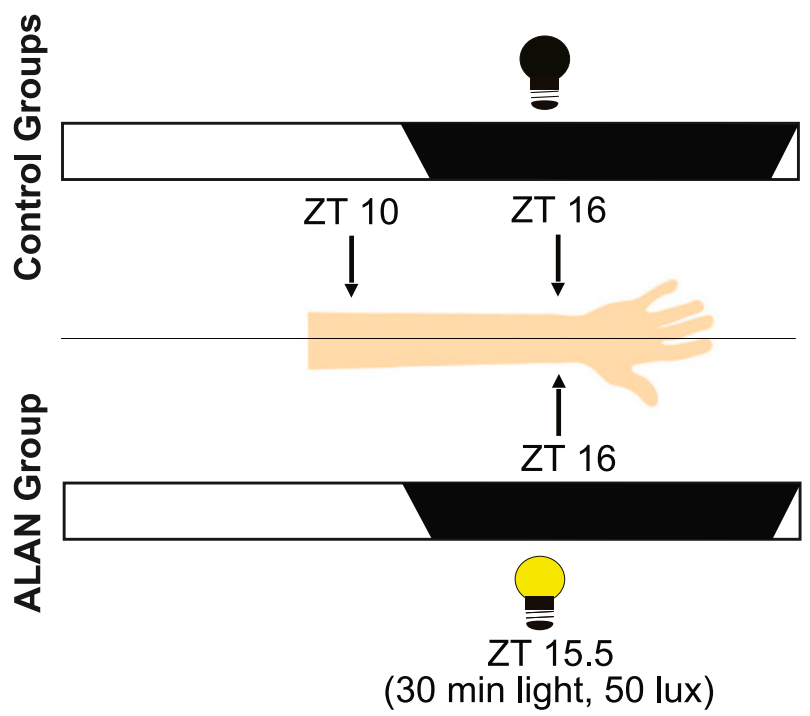

FIGURE 1. Female Aedes aegypti mosquito biting behavior tested using a human host blood-feeding assay. (A) Standard photoschedule for the Ae. aegypti mosquitoes. Zeitgeber time (ZT) 12 = time of lights off; ZT24/0 occurs at the end of 1 hour dawn transition. (B) Method for testing the effect of 50 lux white light presented during the night (starting at ZT15.5 and ending at ZT16) on biting activity (tested at ZT16). Control biting assays occurred during the daytime (ZT10) and at night (ZT16) 2 hours before and 4 hours after the time of lights off/onset of night, respectively. Mosquitoes were assayed for blood feeding for 6 minutes in the dark. White-black bar represents the environmental light-dark (LD) cycle. Arrows directly below or above the LD bar indicate the time of blood meal (exposure to human arm). This figure appears in color at www.ajtmh.org.

Our prediction was that the ALAN group would show a raised propensity to bite the human as compared with the nighttime control group. The results of the study were clear (one-factor analysis of variance (ANOVA), $F_{2,12}=10.24, P=0.0025$, with post hoc Tukey tests; Figure 2, Supplemental Table). Of the mosquitoes assessed during the daytime at ZT10, $82 \%$ of mosquitoes fed. Of the control mosquitoes assessed during the night at ZT16 and maintained under their normal photoschedule, $29 \%$ blood fed. These results showing a large difference in biting behavior between day and night $(P<0.01)$ were predicted from the normal 24-hour diel biting profile of the mosquito as previously documented for Ae. aegypti. In the ALAN group, the propensity to blood feed was at $59 \%$ (Figure 2). Furthermore, no significant difference was observed between the biting propensities of ALAN-treated mosquitoes and the daytime controls. The results of this study reveal a significant elevation in the propensity of the Ae. aegypti mosquito to bite a human host at night following exposure to light at night $(P<0.05)$. This elevation represents a doubling of the biting activity as compared with the activity in the nighttime control group.

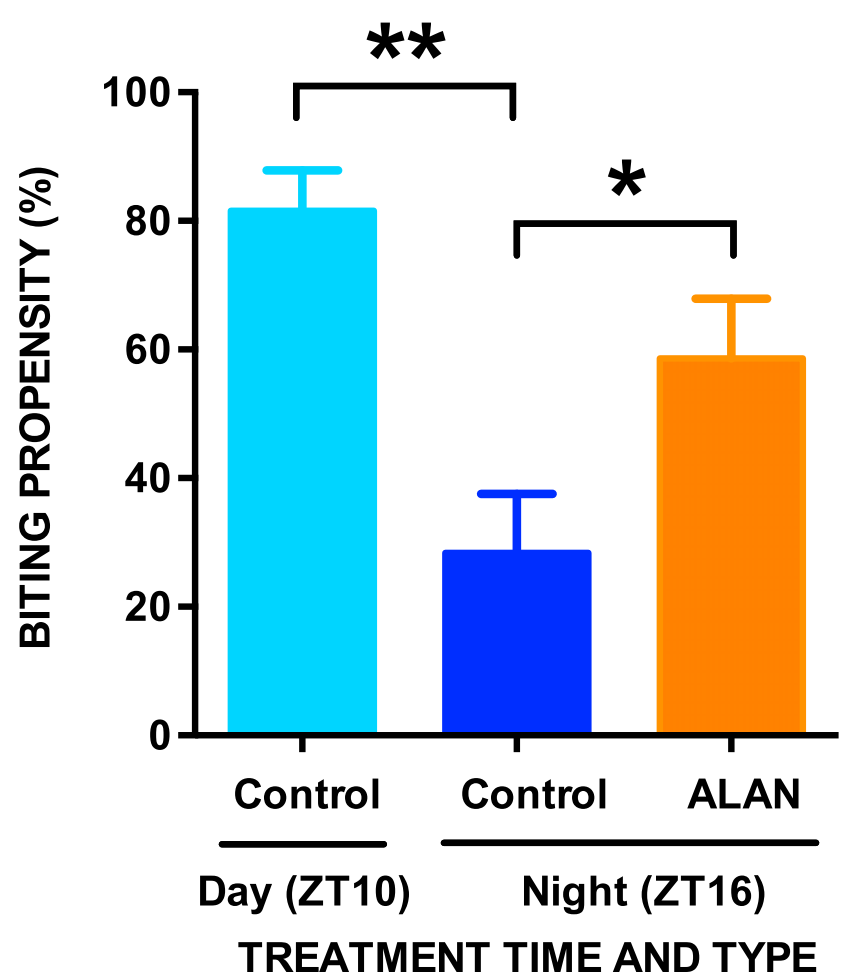

FIGURE 2. Aedes aegypti mosquitoes exposed to light during the night exhibit increased biting activity. Mosquito biting behavior is increased after receiving 30 minutes of light at night. Blood meals were offered at Zeitgeber time (ZT) 10 and ZT16 in the dark, following a normal exposure to the light-dark cycle (controls). One-way ANOVA followed by post hoc tests $\left({ }^{*} P<0.05\right.$ and $\left.{ }^{\star *} P<0.01\right)$. Values shown are mean \pm the standard error of the mean. This figure appears in color at www.ajtmh.org.

The observation of a large increase in biting activity induced by ALAN raises several questions and considerations. One limitation of the study design was that the mosquitoes in the ALAN group were assessed in the dark, similar to the other two control groups. The design of the experiment was to allow for assessment of the mosquitoes under identical environmental conditions. It might be interesting to assess both the daytime control group and the ALAN group in the light, and the nighttime control group in the dark. We predict in this situation measuring an even greater increase in biting activity in the ALAN group versus the nighttime control group. The specific design of this 30-minute pulse then feed protocol ensures that we are testing for the immediate or direct effects of ALAN on the biting behavior. In theory, a phase shift of the intrinsic circadian clock of the mosquito could change the temporal profile of the 24-hour biting preference rhythm. ${ }^{11}$ The clock in other organisms including insects has been shown to be reset by light within 2-3 hours but not at 1 hour after the start of a precisely timed photic stimulus. ${ }^{17,18}$ Therefore, a shift of the circadian clock would not explain the increase of biting that is observed immediately after the 30-minute light treatment in the ALAN group. A shift of the clock might, however, contribute to the changes observed after two or more hours following photic treatment, but we deliberately did not test for this possibility. Lights-on or lights-off can produce a flight activity "startle response," prominently observable at the start and end of the photoschedule when mosquitoes are subjected to a LD cycle with abrupt transitions. ${ }^{4,11,14,15}$ It is plausible that this effect might have influenced the behavior of 
mosquitoes in the current study, specifically in the ZT10 and ALAN groups. These mosquitoes were subjected to a change in light exposure from light to darkness immediately before their blood-feeding assessment. However, as this occurred simultaneously with the other environmental disturbances that transpire as part of the assay, that is, moving the container holding the mosquitoes and exposure to a human host, it is unlikely to have adversely impacted the assessment of biting. Furthermore, it might be expected that as part of humanbased light pollution, wild mosquitoes are exposed to such comparable transitions in illuminance. The intensity of light chosen in this study of 50 lux was based on upper end of the illuminance observed for light pollution at night. It might be predicted that an increase in illuminance or a shift in its color spectrum (wavelength) would alter the effect, ${ }^{10,11}$ but this would have to be tested empirically.

The results of this study highlight the potential impact of ALAN on wild populations of Ae. aegypti mosquitoes, and therefore the increased occurrence of disease transmission. Although primarily a daytime biting vector, nocturnal biting has occasionally been noted in Ae. aegypti. ${ }^{19,20}$ As nocturnal biting was observed in urban Trinidad, but not in rural areas, it was hypothesized that artificial light could be a factor driving this behavior. ${ }^{19}$ The discovery that light increases biting in the diurnal Ae. aegypti mosquito contrasts with our previous work in the nocturnal malaria vector, Anopheles gambiae. We and others have demonstrated light at night suppresses $A n$. gambiae biting behavior. ${ }^{11,21}$ Discovering that nocturnal light stimulates Ae. aegypti blood feeding would suggest that artificial light may be increasing the risk of arboviral disease transmission by increasing the biting rate of the vector, and thus would be of vital epidemiological importance in understanding the risk of disease transmission. This is particularly so for Ae. aegypti because of its anthropophilic peri-domestic behavior, making it likely to be routinely subjected to light pollution as it localizes within the vicinity of human habitation. ${ }^{4}$ Interestingly, nocturnal biting by $A e$. aegypti would also suggest that bed nets, specifically deployed against nocturnal biting insects and especially anopheline malaria-transmitting mosquitoes, may provide some protection against the yellow fever/dengue/Zika mosquito. Rapid urbanization and the peridomestic nature of $A$ e. aegypti mean that this vector species will frequently encounter ALAN that may in turn modulate its behavior.

Received July 20, 2020. Accepted for publication September 12, 2020.

Published online October 15, 2020.

Note: Supplemental table appears at www.ajtmh.org.

Financial support: L. F. L. was funded by a University of Notre Dame (UND) College of Science Summer Undergraduate Research Fellowship, S. S. C. R. was funded by the NIAID (Contract No. HHSN272201400029C), and G. E. D. supported by UND and Eck Institute for Global Health.

Authors' addresses: Samuel S. C. Rund, Laura F. Labb, Owen M. Benefiel, and Giles E. Duffield, Department of Biological Sciences, Galvin Life Science Center, University of Notre Dame, Notre Dame, IN, and Eck Institute for Global Health, University of Notre Dame, Notre Dame, IN, E-mails: srund@nd.edu, llabb@alumni.nd.edu, obenefiel@ gwu.edu, and duffield.2@nd.edu.

\section{REFERENCES}

1. World Health Organization, 2020. Vector-borne Diseases. Geneva, Switzerland: WHO. Available at: https://www.who.int/ news-room/fact-sheets/detail/vector-borne-diseases. Accessed March 2, 2020.

2. Leta S, Beyene TJ, De Clercq EM, Amenu K, Kraemer MUG, Revie CW, 2018. Global risk mapping for major diseases transmitted by Aedes aegypti and Aedes albopictus. Int $J$ Infect Dis 67: 25-35.

3. Chadee DD, 1988. Landing periodicity of the mosquito Aedes aegypti in Trinidad in relation to the timing of insecticidal spacespraying. Med Vet Entomol 2: 189-192.

4. Clements AN, 1999. The Biology of Mosquitoes, Volume II Sensory Reception and Behaviour. Wallingford, Oxfordshire, UK: CABI Publishing.

5. Cinzano P, Falchi F, Elvidge CD, 2001. The first World Atlas of the artificial night sky brightness. Mon Not $R$ Astron Soc 328: 689-707.

6. Longcore T, Rich C, 2004. Ecological light pollution. Front Ecol Environ 2: 191-198.

7. Swaddle JP et al., 2015. A framework to assess evolutionary responses to anthropogenic light and sound. Trends EcolEvol30: 550-560.

8. Hölker F, Wolter C, Perkin EK, Tockner K, 2010. Light pollution as a biodiversity threat. Trends Ecol Evol 25: 681-682.

9. el Jundi B, Warrant EJ, Byrne MJ, Khaldy L, Baird E, Smolka J, Dacke M, 2015. Neural coding underlying the cue preference for celestial orientation. Proc Natl Acad Sci USA 112: 11395-11400.

10. Longcore T, Aldern HL, Eggers JF, Flores S, Franco L, HirshfieldYamanishi E, Petrinec LN, Yan WA, Barroso AM, 2015. Tuning the white light spectrum of light emitting diode lamps to reduce attraction of nocturnal arthropods. Phil Trans R Soc B Biol Sci 370: 20140125

11. Sheppard AD, Rund SSC, George GF, Clark E, Acri DJ, Duffield GE, 2017. Light manipulation of mosquito behaviour: acute and sustained photic suppression of biting activity in the Anopheles gambiae malaria mosquito. Parasit Vectors 10: 255.

12. Rund SS, Gentile JE, Duffield GE, 2013. Extensive circadian and light regulation of the transcriptome in the malaria mosquito Anopheles gambiae. BMC Genomics 14: 218.

13. Saunders DS, Steel CGH, Vafopoulou X, Lewis RD, 2002. Insect Clocks. Amsterdam, The Netherlands: Elsevier.

14. Taylor B, Jones MD, 1969. The circadian rhythm of flight activity in the mosquito Aedes aegypti (L.). The phase-setting effects of light-on and light-off. J Exp Biol 51: 59-70.

15. Gentile C, Rivas GB, Meireles-Filho AC, Lima JB, Peixoto AA, 2009. Circadian expression of clock genes in two mosquito disease vectors: cry2 is different. J Biol Rhythms 24: 444-451.

16. Kang DS, Barron MS, Lovin DD, Cunningham JM, Eng MW, Chadee DD, Li J, Severson DW, 2018. A transcriptomic survey of the impact of environmental stress on response to dengue virus in the mosquito, Aedes aegypti. PLoS Negl Trop Dis 12: e0006568.

17. Best J, Maywood E, Smith K, Hastings M, 1999. Rapid resetting of the mammalian circadian clock. J Neurosci 19: 828-835.

18. Pittendrigh C, 1979. Some functional aspects of circadian pacemakers. Suda M, Hayaishi O, Nakagawa H, eds. Biological Rhythms and Their Central Mechanism. Amsterdam, The Netherlands: Elsevier Press, 3-12.

19. Chadee DD, Martinez R, 2000. Landing periodicity of Aedes aegypti with implications for dengue transmission in Trinidad, West Indies. J Vector Ecol 25: 158-163.

20. Teesdale C, 1955. Studies on the bionomics of Aedes aegypti (L.) in its natural habitats in a coastal region of Kenya. Bull Ent Res 46: 711-742.

21. Das S, Dimopoulos G, 2008. Molecular analysis of photic inhibition of blood-feeding in Anopheles gambiae. BMC Physiol 8: 23. 